\section{Cycling back to the beginning}

\author{
Michael Whitfield
}

The Carbon Cycle and Atmospheric $\mathrm{CO}_{2}$ : Natural Variations Archean to Present. Edited by E.T. Sundquist and W.S. Broecker. American Geophysical Union: 1985. Pp.627. \$28.

IT Is refreshing - although somewhat disconcerting - for someone involved in the investigation of the contemporary global $\mathrm{CO}_{2}$ cycle and its possible climatic effects to view the problem from a much broader perspective. This volume jolts the reader into considering such a wider view by presenting a stimulating potpourri of ideas on the direction, cause and consequences of natural fluctuations in atmospheric $\mathrm{CO}_{2}$ levels over the past $3.5 \times 10^{9} \mathrm{yr}$.

There are more than 40 papers, replete with useful data and providing intriguing insights into the response of different feedback systems to perturbations in the global carbon cycle over timescales varying from tens of thousands to hundreds of millions of years. The papers themselves have been edited thoroughly, and the text is clearly presented and well illustrated. The overall structure, too, is well conceived, in that the contents list is organized into five sections which move progressively further back in time. The first section is intended to link present-day concerns to the geological perspective while subsequent sections take the reader in turn through the last glaciation, the Pleistocene, the Cenozoic, and finally the Phanerozoic and Precambrian. There is a wealth of information here which, however, is unfortunately difficult to dig out because the book has no index and the papers within each section do not necessarily follow a logical sequence. Furthermore the sections identified in the contents list are not explicitly identified in the text, so that the browser finds himself slipping unwittingly from one timescale to another.

The first three sections cover the past 5 $\times 10^{5} \mathrm{yr}$ and mainly deal with the use of the carbon and oxygen isotope records in ice corcs and in deep-sea sediments to elucidate the role of the oceans in controlling fluctuations in atmospheric $\mathrm{CO}_{2}$ levels. Only one paper deals with the influence of the terrestrial biota on carbon storage. There are many absorbing accounts here of the potential and pitfalls of isotopic techniques for estimating atmospheric $\mathrm{CO}_{2}$ levels, ice volumes, palaeoproductivity and palaeocirculation patterns. Over this timescale, orbital variations appear to trigger changes in atmospheric $\mathrm{CO}_{2}$ levels which are, in turn, followed by changes in ice volume.

During the Cenozoic $\left(10^{8} \mathrm{yr}\right)$, processes

such as volcanism, tectonic movement, continental weathering and organic carbon burial begin to assume a dominant role. In addition to isotopic clues, crises in the biological record characterized by mass extinctions provide useful markers against which to assess the interaction between the biota and the atmosphere. Surprisingly, only the Cretaceous/Tertiary event is considered here, although the discussion is spiced by the presentation of two conflicting hypotheses (planetismal impact and mantle outgassing).

Geochemical models for $\mathrm{CO}_{2}$ control in the Phanerozoic are covered in some detail in the final section, but the treatment of the Precambrian is rather sketchy so that only 15 per cent of the book as a whole considers the first 99.75 per cent of geological time! The overall picture suggested is of a punctuated fall from atmospheric $\mathrm{CO}$, concentrations one hundred

\section{Uncertain alliance}

\section{Charles Tanford}

The Physical Chemistry of Membranes: An Introduction to the Structure and Dynamics of Biological Membranes. By Brian L. Silver. Allen \& Unwin: 1985. Pp.396. £45, $\$ 60$.

To present a concise account of all physico-chemical aspects of membranes in a single book, suitable for students and beginning researchers, is difficult because membrane physical chemistry comprises two quite different bodies of knowledge. One centres on the structure and molecular dynamics of the membrane, and includes the physical methods (nuclear magnetic resonance, neutron scattering etc.) used for structural investigations but should also include some account of the molecular interactions that hold the membrane together. The other part centres on the physical chemistry of membrane function, and is chiefly thermodynamic and kinetic in its approach to questions of membrane potential, permeability, and carrier or protein mediated transport. Conventional wisdom suggests that these two fields might be better tackled by separate authors in separate books.

Dr Silver's book attempts to include all these topics but regrettably cannot be recommended for either structural or functional information. Its broad organization is good: 18 chapters with appropriate titles. Within each chapter, unfortunately, there is insufficiency, often chaos, sometimes error, and always a flippant haphazardness as to what is selected for discussion and what is omitted, who is cited and who is not. The entire book has only 378 pages of text which includes 125 figures, many of them full page. times the present level $3 \times 10^{9}$ yr ago, through a value approximately ten times the present level $10^{8} \mathrm{yr}$ ago, to a preindustrial value only 80 per cent of the present level. The burial and preservation of organic carbon has played a primary role in this decline - a factor which geochemical modellers are now taking into account.

This is a fascinating collection of papers which will repay the effort required for specialist readers to pick their way through such a variety of information. The cost is very reasonable, and one can hope that many individuals will buy the book and use it to help them place the current ferment in atmospheric $\mathrm{CO}$, research into a proper perspective.

Michael Whitfield is a Senior Principal Scientific Officer at the Marine Biological Association of the UK, The Laboratory, Citadel Hill, Plymouth PLI 2PB, UK.

The better chapters are one that deals with order and disorder, as seen by nuclear magnetic resonance and electron spin resonance; one on intermolecular forces, which includes a mathematically detailed section on van der Waals' forces; and one on fluctuations, which includes an introduction to stochastic processes. Order/ disorder is in part explained by picturesque images (a box of uncooked spaghetti, a pile of sleepy earthworms) that may be helpful to some students.

The poorer chapters are those that deal with solution physical chemistry (thermodynamics, kinetics, diffusion, membrane potential etc.). For example, the "celebrated Nernst equilibrium equation" is derived, properly limited to boundaries with the same solvent on both sides, only to be applied on the very next page to the equilibrium distribution of an ion between the membrane interior and the adjacent solution.

In the next chapter, on passive transport, the author argues as to whether or not diffusion can be regarded as arising from the action of a force. His conclusion is not clear but a subsequent parenthetical statement - "The theoretically inclined will perhaps remember that the diffusion coefficient is related to the autocorrelation function for the random force beating on the molecule" - implies perhaps that diffusion can be regarded in this way. The following chapter is centred on Eyring's absolute rate theory for diffusion, but misrepresents the basic ideas behind the theory by confusing energy and free energy of the activated state.

Silver has a lively style and an instinct for apt imagery. Combination of these assets with more careful theoretical analysis could produce a truly useful book.

Charles Tanford is J.B. Duke Professor of Physiology at Duke University, Box 3709-P, Durham, North Carolina 27710, USA. 